\title{
Case Report \\ Rhizopus microsporus var. rhizopodiformis sinus-orbital zygomycosis in an immunosuppressed patient: successful treatment with posaconazole after a complicated clinical course
}

\section{Correspondence \\ D. Stark \\ dstark@stvincents.com.au}

Received 13 November 2006

Accepted 10 January 2007
D. Stark, ${ }^{1}$ S. Milliken, ${ }^{2}$ D. Marriott ${ }^{1}$ and J. Harkness ${ }^{1}$

${ }^{1}$ Department of Microbiology, St Vincent's Hospital, Sydney, Australia
${ }^{2}$ Department of Haematology, St Vincent's Hospital, Sydney, Australia

A case of sinus-orbital Rhizopus microsporus var. rhizopodiformis infection in a patient with graft versus host disease following allogeneic blood stem cell transplantation is reported.

Commercially available pea straw compost used for gardening was suspected to be the source of the infection. After an initial relapse, treatment with surgical debridement, liposomal amphotericin $B$ and posaconazole was successful.

\section{Case report}

A 42-year-old man presented with a 14 day history of worsening sinus congestion, facial swelling and pain. The patient reported that his symptoms commenced after mulching his garden with pea straw. Seven months earlier he had undergone a human leukocyte antigen matched, sibling donor, allogeneic peripheral blood stem cell transplant for chronic lymphocytic leukaemia (CLL). CLL had been diagnosed 4 years prior to the transplant and was treated initially with oral cyclophosphamide and prednisone. Two intravenous cycles of conventional dose fludarabine and cyclophosphamide were given to control progressive CLL a month before transplant. Transplant conditioning was high-dose cyclophosphamide (120 mg $\mathrm{kg}^{-1}$ ) and fractionated total body irradiation, $12 \mathrm{~Gy}(\mathrm{TBI})$ and graft versus host disease (GvHD) prophylaxis consisted of cyclosporin, methotrexate and prednisone. Acute GvHD occurred 20 days post-transplant with stage 3 skin and stage 2 gut and liver involvement (grade III GvHD). GvHD was treated with pulsed intravenous methylprednisolone (total dose $3.5 \mathrm{~g}$ ), then prednisone $1 \mathrm{mg} \mathrm{kg}^{-1}$ b.i.d. and mycophenolate mofetil in addition to continuing cyclosporin. Other complications were cytomegalovirus viraemia occurring 7 weeks post-transplant and detected by surveillance PCR testing. The viraemia resolved with intravenous foscarnet therapy. Haemorrhagic cystitis developed 12 weeks post-transplant and improved with bladder irrigation and symptomatic therapy. Antifungal prophylaxis consisted of oral itraconazole $400 \mathrm{mg}$ daily with blood level monitoring.
The patient was initially treated for sinusitis with intravenous antibiotics at a rural hospital. Sinus washout was performed after $72 \mathrm{~h}$ as his condition was worsening and pathological review of washout material demonstrated copious fungal elements. He was urgently transferred to the transplant centre, where treatment was commenced with liposomal amphotericin $5 \mathrm{mg} \mathrm{kg}^{-1}$ per day. Fifteen days after the initial presentation, MR and CT scans demonstrated extensive sinusitis with extension into the inferior left orbit and soft tissues of the left face. CT of the chest and abdomen demonstrated multiple cavitating pulmonary nodules. Sinus washout and biopsies of the sinus mucosa and a lung nodule confirmed invasive fungal infection with the morphological appearance of a zygomycete infection. Subsequent culture identified Rhizopus microsporus. Further surgery with sinus debridement and excision was performed. After 8 days of liposomal amphotericin B therapy there was no clinical or radiological response and the dose was increased to $7.5 \mathrm{mg} \mathrm{kg}^{-1}$ and subsequently $10 \mathrm{mg} \mathrm{kg}^{-1}$ per day after a further 4 days without clinical response. After 25 days therapy the patient remained unwell and serial CT scanning demonstrated no improvement. Posaconazole ( $200 \mathrm{mg}$ q.i.d. orally) was commenced through a compassionate access scheme, the only mechanism of obtaining the drug in Australia at the time. Liposomal amphotericin B was continued but the dose was reduced and subsequently ceased because of nephrotoxicity and lack of apparent clinical response (total dose $>12 \mathrm{~g}$ ). Intravenous gamma-globulin was given to correct hypogammaglobulinaemia and immunosuppressive therapy was reduced.

Within 7 days of commencing posaconazole there was significant clinical improvement with reduction of facial 
pain and swelling. A CT scan performed 9 days after commencement of posaconazole demonstrated stabilization of disease and the patient was well enough to be discharged from hospital, continuing therapy as an outpatient. Four weeks later the patient developed a left orbital cellulitis with an underlying orbital abscess associated with neutropenia related to mycophenolate mofetil therapy. This was surgically drained and the neutropenia resolved with granulocyte-colony stimulating factor therapy and withdrawal of mycophenolate mofetil. Cultures grew group D streptococcus and the infection responded to intravenous antibiotics. No fungi were isolated.

Four months later the patient was readmitted with recurrent facial pain, and further surgery demonstrated extensive necrotic tissue. Frontal and left maxillary sinuses and the left orbital floor were resected. Biopsies revealed necrotic tissue containing fungal hyphae but fungal cultures were negative. Two weeks after surgery the patient experienced an acute, life-threatening epistaxis due to erosion of the right internal carotid artery. The artery was subsequently embolized without further complication.

Six months after commencing posaconazole the patient was well and asymptomatic, and his maxillary sinus had closed. A CT scan demonstrated no further bony erosion of the sinuses and improvement in the pulmonary lesions was noted.

Further sinus surgery and debridement of necrotic tissue was performed 7 and 9 months after commencing therapy. Biopsies again revealed necrotic tissue containing fungal hyphae, with fungal cultures remaining negative. CT scanning of sinuses and chest showed that they remained stable.

One year after infection the patient remains well and continues posaconazole therapy.

\section{Microbiology}

Sinus biopsies and sinus swabs were inoculated onto blood agar (Oxoid) and chocolate agar (Oxoid) at $37{ }^{\circ} \mathrm{C}$ in a $5 \%$ $\mathrm{CO}_{2}$ atmosphere and Sabouraud agar (Oxoid) at $30{ }^{\circ} \mathrm{C}$ in air. After 3 days incubation a dark-greyish brown mould was growing on all plates. The organism was provisionally identified as a Rhizopus species and was referred to the Mycology Laboratory at the Royal Women's and Children's Hospital, Adelaide, where it was identified as $R$. microsporus var. rhizopodiformis based on traditional phenotypic methods. Susceptibility testing was performed in accordance with Clinical and Laboratory Standards Institute guidelines, with the isolate being sensitive to amphotericin B, and resistant to 5-fluorocytosine, fluconazole, itraconazole, ketaconazole and voriconazole. Unfortunately no in vitro susceptibility data were obtained for posaconazole. Samples of the peat straw compost were obtained and cultured onto Sabouraud agar at $30{ }^{\circ} \mathrm{C}$ in air. This environmental sample grew a rapidly growing mould that was morphologically identified as a Rhizopus species, in addition to environmental saprophytic fungi.

Genomic DNA was extracted from the clinical cultures using a QIAamp DNA Mini kit (QIAGEN) and DNA amplification of the internal transcribed spacer (ITS) regions of the rRNA gene complex was followed by sequencing. The PCR assay was performed using two oligonucleotide fungal primers (ITS1 and ITS4) targeting the ITS regions of the rRNA gene complex, as described previously (Henry et al., 2000). The PCR products were then purified using the QIAquick PCR Purification kit (QIAGEN) as per the manufacturer's instructions. The PCR product was sequenced directly in both directions using the ITS1 and ITS4 primers on an ABI Prism 3730 automated sequencer at the SUPAMAC facility (Royal Prince Alfred Hospital, Sydney). The sequences were then compared to those available in the GenBank databases using the BLASTN program run on the National Center for Biotechnology Information server (http://www.ncbi.nlm. nih.gov/BLAST/).

The ITS gene sequences generated from both the clinical and environmental samples were identical and showed $100 \%$ similarity with the sequence of the ITS gene from the CBS 343.29 strain of $R$. microsporus var. rhizopodiformis (GenBank accession no. AY243958).

\section{Discussion}

R. microsporus is a thermophilic zygomycete with a worldwide distribution, and is saprophytic, living in soil, air and decaying matter and growing well on substrates such as fruits, cereals and breads (Ribes et al., 2000). Among the four known varieties, only $R$. microsporus var. microsporus and $R$. microsporus var. rhizopodiformis have been reported to cause infections in humans. $R$. microsporus var. rhizopodiformis may be a cause of human zygomycosis with over 20 cases reported in the literature (Roden et al., 2005), particularly the cutaneous and gastrointestinal forms (Eucker et al., 2001). Cutaneous disease (Neame \& Rayner, 1960; West et al., 1995; Ribes et al., 2000; Eucker et al., 2001), gastric zygomycosis and fatal fungal peritonitis (Neame \& Rayner, 1960; Nakamura et al., 1989; Kimura et al., 1995) associated with $R$. microsporus var. rhizopodiformis have been documented. A single case of pulmonary (Maniwa et al., 2002) and sinoorbital (Rosenberger et al., 1983) infection has also been reported. Most infections are opportunistic with patients having severe underlying disease conditions and/or immunosuppression. Host risk factors include diabetes, leukaemia, bone marrow transplantation, immunosuppressive chemotherapy, broad-spectrum antibiotic use and breakdown of the cutaneous barrier (Eucker et al., 2001; Nucci, 2003). Mortality rates associated with infection are 70-100\% (Nucci, 2003).

The ITS regions have been shown to be species-specific for the differentiation of a wide variety of fungal pathogens, 
including zygomycetes, and offer a rapid identification (Henry et al., 2000; Iwen et al., 2002, 2005). The ITS regions are located between the $18 \mathrm{~S}$ and $28 \mathrm{~S}$ rRNA genes and have approximately 100 copies per fungal genome, which increases sensitivity of the assay. As the recovery of zygomycetes from tissues can be difficult, with fungal growth often hindered by specimen processing, PCR and direct sequencing techniques may have an important role in species identification.

There are currently no valid typing techniques for any species of zygomycete and although the sequence data generated at the ITS of the rRNA gene complex regions from both the clinical and environmental isolates showed $100 \%$ similarity, sequencing of the ITS region is not suitable for typing isolates of Rhizopus. Although there is an epidemiological suspicion of the pea straw as an environmental source of infection, the molecular technique used is not sufficiently discriminatory. However, it is very likely that the source of infection originated from the pea straw. This is consistent with the fact that $R$. microsporus var. rhizopodiformis is a saprophytic fungus which is most commonly found in soils and decaying matter.

Zygomycete infections have a poor outcome when complicating blood or bone marrow stem cell transplantation (Ribes et al., 2000). In our patient, the long clinical course and need for repeated surgery to excise necrotic tissue highlight the difficulties in treating this infection. There was no response to a standard dose of liposomal amphotericin B, and dose escalation caused renal toxicity. It is likely that the combination of repeated surgery, reduction of immunosuppression and posaconazole were the important factors in achieving control of the infection. This is consistent with the therapeutic recommendations of other authors (Ribes et al., 2000). The repeated findings in this patient of fungal hyphae in necrotic tissue, even without positive culture, suggests that long-term therapy with posaconazole as well as regular surgical examination and debridement are needed for a successful outcome. Posaconazole was well tolerated without any apparent toxicity.

This is only the second reported case of a clinical isolate of $R$. microsporus var. rhizopodiformis confirmed by molecular techniques. The ITS region has not been previously used for identification of the organism from a clinical source. This report highlights the usefulness of ITS sequence analysis for identification of uncommon pathogenic species.

\section{Acknowledgements}

The authors thank Associate Professor David Ellis for the morphological identification and susceptibility testing of the fungus.

\section{References}

Eucker, J., Sezer, O., Graf, B. K. \& Possinger, K. (2001). Mucormycoses. Mycoses 44, 253-260.

Henry, T., Iwen, P. C. \& Hinrichs, S. H. (2000). Identification of Aspergillus species using internal transcribed spacer regions 1 and 2. $J$ Clin Microbiol 38, 1510-1515.

Iwen, P. C., Hinrichs, S. H. \& Rupp, M. E. (2002). Utilisation of the internal transcribed spacer regions as molecular targets to detect and identify human fungal pathogens. Med Mycol 40, 87-109.

Iwen, P. C., Freifeld, A. G., Sigler, L. \& Tarantolo, S. R. (2005). Molecular identification of Rhizomucor pussillus as a cause of sinoorbital zygomycosis in a patient with acute myelogenous leukemia. $J$ Clin Microbiol 43, 5819-5821.

Kimura, M., Udagawa, S. R., Toyazaki, N., limori, M. \& Hashimoto, S. (1995). Isolation of Rhizopus microsporus var rhizopodiformis in the ulcer of human gastric carcinoma. J Med Vet Mycol 33, 137-139.

Maniwa, K., Tanaka, E., Taguchi, Y., Oida, K., Inoue, T., Kato, T., Sakuramoto, M., Maeda, Y., Terada, K. \& Aihara, M. (2002). A case of abrupt pulmonary infection by Rhizopus microsporus var rhizopodiformis during treatment for bronchial asthma. Kansenshogaku Zasshi 76, 396-399.

Nakamura, M., Weil, W. B., Jr \& Kaufman, D. B. (1989). Fatal fungal peritonitis in an adolescent on continuous ambulatory peritoneal dialysis: association with deferxamine. Pediatr Nephrol 3, 80-82.

Neame, P. \& Rayner, D. (1960). Mucormycosis, a report on twentyone cases. Arch Pathol 70, 261-268.

Nucci, M. (2003). Emerging moulds: Fusarium, Secedosporium and Zygomycetes in transplant recipients. Curr Opin Infect Dis 16, 607-612.

Ribes, J. A., Vonover-Sams, C. L. \& Baker, D. J. (2000). Zygomycetes in human disease. Clin Microbiol Rev 13, 236-301.

Roden, M. M., Zaoutis, T. E., Buchanan, W. L., Knudsen, T. A., Sarkisova, T. A., Schaufele, R. L., Sein, M., Sein, T., Chiou, C. C. \& other authors (2005). Epidemiology and outcome of zygomycosis: a review of 929 reported cases. Clin Infect Dis 41, 634-653.

Rosenberger, R. S., West, B. C. \& King, J. W. (1983). Survival from sino-orbital mucormycosis due to Rhizopus rhizopodiformis. Am J Med Sci 286, 25-30.

West, B. C., Oberle, A. R. \& Kwon-Chung, K. J. (1995). Mucormycosis caused by Rhizopus microsporus var. microsporus: cellulitis in the leg of a diabetic patient cured by amputation. J Clin Microbiol 33, 3341-3344. 